\title{
UNIDADES DE ACTUACIÓN URBANÍSTICA O DE GESTIÓN: CASOS DE APLICACIÓN EN TRES PLANES PARCIALES DE BOGOTÁ D. C.
}

\author{
URBAN ACTION OR MANAGEMENT UNITS: APPLICATION CASES IN THREE \\ PARTIAL PLANS OF BOGOTÁ D.C.
}

Tadeo Humberto Sanabria Artunduaga*

John Fredy Ramírez Ríos ${ }^{\text {*** }}$

Jhonny Giovanny Puentes Estévez***

Recibido: 15 de agosto de 2017

Aceptado: 10 de noviembre de 2017

\section{Resumen}

Se presentan los resultados de la comparación de tres planes parciales formulados para el desarrollo y renovación urbana de diferentes zonas de la ciudad de Bogotá. Se hace énfasis en las dos formas de aplicación de los instrumentos de gestión: unidades de actuación urbanística y unidades de gestión. Se analizan las circunstancias específicas que explican la aplicación de cada uno de estos instrumentos en los planes comparados; igualmente, se describe cómo la primera figura opera cuando existen propietarios renuentes, y cómo la segunda opera si hay consenso pleno para la asignación de cargas y beneficios entre estos.

Palabras clave: gestión territorial, unidades de actuación urbanística, unidades de gestión, plan parcial.

\begin{abstract}
The results of the comparison of three partial plans formulated for the development and urban renewal of different areas of the city of Bogota are presented. Emphasis is placed on the two forms of application of management instruments: urban action units and management units. The specific circumstances that explain the implementation of each of these tools in the comparative plans are analyzed. It also describes how the first figure operates when there are reluctant owners, and how the second works if there is full consensus for the allocation of charges and benefits among these.
\end{abstract}

Keywords: territorial management, urban action units, management units, partial plan.

\footnotetext{
* Arquitecto, magíster en Ordenamiento Urbano y Regional, magíster en Gestión y Valoración Urbana, doctor en Gestión y Valoración Urbana y Arquitectónica. Docente investigador, grupo de investigación Prospectiva Urbano Territorial, línea de investigación Ciudades Competitivas, Facultad de Educación Permanente y Avanzada, Fundación Universidad de América, Bogotá, Colombia. ORCID: http://orcid.org/0000-0003-2517-2629. Tadeo.sanabria @ investigadores.uamerica.edu.co

** Sociólogo, magíster en Urbanismo. Docente investigador, Grupo de investigación Prospectiva Urbano Territorial, línea de investigación Ciudades Competitivas, Facultad de Educación Permanente y Avanzada, Fundación Universidad de América, Bogotá, Colombia. ORCID: http://orcid.org/0000-0002-3006-7228. john.ramirez@ investigadores. uamerica.edu.co

${ }^{* * \star}$ Arquitecto, especialista en Planeación Territorial, Bogotá, Colombia. giovannypuentesestevez@gmail.com
} 


\section{INTRODUCCIÓN}

La Ley 388 de 1997 o Ley de Desarrollo Territorial (LDT) obliga a los municipios colombianos a formular un plan de ordenamiento territorial (POT). Según esta ley, el POT contiene la visión, políticas, estrategias, clasificaciones y tratamientos de suelo más generales que deben ser aplicados sobre un territorio municipal. Además, la Ley contempla un instrumento para la planificación de escala intermedia en zonas específicas de uso urbano, denominado Plan Parcial (PP) (Tarchópulos, 2006). Según Maldonado, Pinilla, Rodríguez y Valencia (2006), el PP:

Es el instrumento más importante del sistema urbanístico colombiano: concreta la articulación entre la planeación y la gestión del suelo; y constituye la base para la gestión asociada de los terrenos y la definición de mecanismos concretos de financiación con base en el suelo. (p. 73)

La LDT introdujo un instrumento adicional de gestión territorial de escala detallada, la Unidad de Actuación Urbanística (UAU). Esta se define como:

El área conformada por uno varios inmuebles, explícitamente delimitada en las normas que desarro1la el plan de ordenamiento que debe ser urbanizada o construida como una unidad de planeamiento con el objeto de promover el uso racional del suelo, garantizar el cumplimiento de las normas urbanísticas y facilitar la dotación con cargo a sus propietarios, de la infraestructura para el transporte, los servicios públicos domiciliarios y los equipamientos colectivos mediante reparto equitativo de las cargas y beneficios. (Art. 39)

Mientras el POT y los PP son instrumentos para el planeamiento, las UAU forman parte del grupo de mecanismos para la gestión de lo planeado. Para reglamentar su aplicación se expidió el Decreto 1507 de 1998, que fue derogado dos años después por el Decreto 2320 de 2000. En este último se aclaró que todo lo concerniente a las UAU ya estaba reglamentado en la LDT. En la Ley se prefiguraron dos escenarios de aplicación de este instrumento de gestión: uno de cooperación, en el que todos los propietarios de un área de planeamiento están de acuerdo con lo que el POT o el PP ha determinado para su área de intervención y forman parte activa de los cambios demandados; y otro de no cooperación, en el que no existe unanimidad alrededor de lo que el POT o el PP demandan para el área de planeamiento. En este último caso, la unidad gestora del POT o del PP en cuestión opera de manera unilateral las UAU.

En el Documento Técnico de Soporte y Cartográfico del Plan Parcial de La Sabana (2014) se indica que una UG se ejecuta cuando "existe acuerdo del $100 \%$ de los propietarios del suelo de la U.A.U. y en los términos definidos por el artículo 19 del Decreto Nacional 2181 de 2006“ (p. 205). En caso contrario, la UAU opera por medio de la constitución de una "entidad gestora o un operador urbano que les permita acompañar y apoyar el proceso de aplicación de los instrumentos normativos para hacer viable la implementación del plan” (p. 205). En este artículo se comparan cómo han operado esos escenarios de aplicabilidad de las UAU o UG en tres planes parciales.

\section{Método}

Este estudio analizó tres planes parciales de renovación urbana: el Plan Parcial de Renovación Urbana La Sabana (PPLS) (Decreto 073 de 2015), que se formuló para un sector de la localidad de Los Mártires y que opera con UAU y UG; el Plan Parcial de Renovación Urbana de San Victorino (PPSV), que fue concebido para operar con UAU y actualmente está pendiente de acto administrativo, y el Plan Parcial La Felicidad (PPLF) (Decreto 147 de 2008), adoptado para el desarrollo de una zona de la localidad de Fontibón, cuya ejecución está en más del 50 \% y opera mediante UG. 
UNIDADES DE ACTUACIÓN URBANISTICA O DE GESTIÓN: CASOS DE APLICACIÓN EN TRES PLANES PARCIALES DE BOGOTÁ D. C.

Tabla 1. Análisis de las fichas de registro de datos de los PP comparados

\begin{tabular}{llll}
\hline \multicolumn{1}{c}{ Tipo } & \multicolumn{1}{c}{ PPLS } & \multicolumn{1}{c}{ PPSV } & \multicolumn{1}{c}{ PPLF } \\
\hline & \multicolumn{1}{c}{ Renovación urbana } & Renovación urbana & $\begin{array}{c}\text { Tratamiento urbano de } \\
\text { desarrollo }\end{array}$ \\
\hline Área $\left(\mathrm{m}^{2}\right)$ & 29258.10 & 147185.84 & 999503.84 \\
Área útil $\left(\mathrm{m}^{2}\right)$ & 15721.30 & 56365.92 & 390866.30 \\
Unidad de actuación aplicada & UAU y/o UG & UAU & UG \\
\# de UAU/UG aplicadas & 6 & 2 & 7 \\
Promotor & Concreta Gestión Urbana S.A.S & Ecourbia S.A.S & Pedro Gómez y Cía \\
Resolución de viabilidad & N.o 1276 & N.o 2465 & N.o 0694 \\
Decreto adopción & $(16-10-16)$ & $(2009)$ & (19-06-15) \\
\hline
\end{tabular}

Fuente: elaboración propia, bajo la recolección de datos en la técnica de análisis documental, basada en Alcaldía Mayor de Bogotá (2014, 2016, 2015).

Los PP escogidos pertenecen a una misma entidad territorial; esto se hizo con la intención de mantener la unidad de análisis para la comparación. Este estudio usa el mismo método de evaluación, que en lo particular hace referencia al marco normativo, pues, aunque los instrumentos de mayor jerarquía tienen influencia y aplicación nacional, en la ejecución de los instrumentos locales existen diferencias de criterio de acuerdo con las características de cada PP.

A partir de esta selección se sustrajo la información más relevante de cada PP, con la intención de detallar cómo ha sido la aplicación y delimitación de los instrumentos de planeación local con los cuales operan, y cómo han operado los instrumentos claves en el desarrollo y la recomposición, revitalización y reconfiguración de algunas áreas en distintos sectores de la capital del país. Se advierte que la operatividad de un PP requiere definitivamente de la operación de una UP de menor jerarquía para que el instrumento PP de mayor jerarquía funcione correctamente.

La información y los datos de estos instrumentos fueron extraídos de los documentos técnicos de soporte (DTS) y el respectivo decreto de adopción (DA). El DA aplica solo para los casos de La Felicidad y La Sabana, ya que San Victorino no cuenta con un acto administrativo proferido por el Distrito Capital.

Finalmente y después de la comparación de acuerdo a los criterios adoptados: a) se coteja los ámbitos y condiciones de actuación de cada tipo de unidad; b) se identifican mediante análisis documental las variables o categorías que permiten su comparación, y c) se reconoce el valor de los componentes que determinan el empleo de cada instrumento para desarrollar la tabla de análisis comparativo en la aplicación de estas UP en los PP.

\section{Resultados}

\section{Plan Parcial de Renovación Urbana "La Sabana"}

El PPLS pretende generar una construcción colectiva en una zona céntrica de la ciudad de Bogotá, sector de interés histórico para la capital, en el que hay edificaciones e infraestructura declaradas bienes de interés cultural y patrimonio arquitectónico. Este proyecto fue adoptado mediante Decreto 073 del 26 de febrero de 2015 y está pendiente de ejecución.

El área a intervenir se ubica al costado norte de la famosa calle 13, entre carreras 18 (futura Av. Mariscal Sucre) y 17 con calle 16; manzanas correspondientes al barrio San Victorino de la Unidad de Planificación Zonal (UPZ) 102 La Sabana, de la localidad de Los Mártires. La zona de 
planificación de este PP tiene un área aproximada de $30000 \mathrm{~m}^{2}$ y una composición de tres manzanas con una base predial de 2.2 hectáreas aproximadamente.
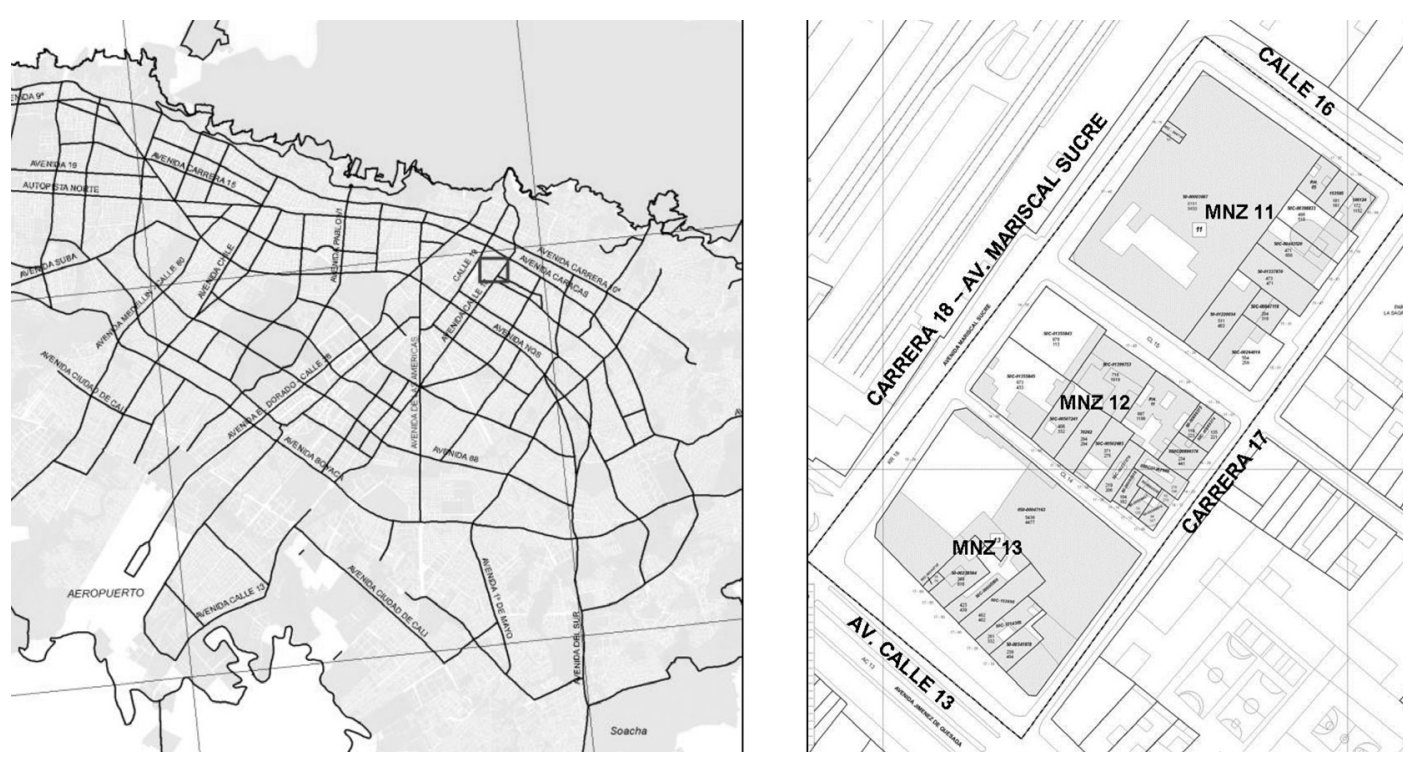

Figura 1. Localización del PPLS. Fuente: Alcaldía Mayor de Bogotá (2014, p 29)

La delimitación del área a intervenir del PPLS tomó como referencia el proceso de crecimiento urbano y la filiación de los rasgos arquitectónicos de algunas edificaciones, como la comprensión del sector en cuanto a los estilos de vida, las costumbres y los usos desarrollados en el entorno más cercano. En total son 34 predios vinculados al proceso de planeación, que pueden ser parte de la unidad gestora y serán beneficiados y/o afectados a la hora de aplicar el reparto equitativo de cargas y beneficios, y que se desarrollará mediante seis unidades de actuación urbanística:

- La manzana 11 y parte de la calle 15 conformarán la U.A.U y/o UG 1 y 2

- La manzana 12 y parte de las calles 14 y 15 conformarán la U.A.U y/o UG 3 y 4.

- La manzana 13 y parte de la calle 14 conformarían la U.A.U y/o UG 5 y 6.

Tabla 2. Ficha de Caracterización del PPLS

\begin{tabular}{|c|c|c|c|c|c|}
\hline \multirow{4}{*}{ BASE PREDIAL } & N.o manzana & Área $\mathbf{m}^{2}$ & $\begin{array}{l}\text { Número de } \\
\text { predios }\end{array}$ & $\begin{array}{l}\text { Índice de } \\
\text { ocupación }\end{array}$ & $\begin{array}{l}\text { Número de } \\
\text { propietarios }\end{array}$ \\
\hline & 11 & 9386.41 & 11 & Más del $90 \%$ & \multirow{3}{*}{50} \\
\hline & 12 & 5545.67 & 16 & $80 \%$ & \\
\hline & 13 & 7307.08 & 7 & $70 \%$ & \\
\hline \multirow{4}{*}{$\begin{array}{c}\text { ÁREAS } \\
\text { EXISTENTES } \mathrm{m}^{2}\end{array}$} & \multicolumn{2}{|l|}{ Área bruta } & \multicolumn{2}{|l|}{29258.10} & \\
\hline & \multicolumn{2}{|l|}{ Malla vial arterial } & \multicolumn{2}{|l|}{2864.93} & \\
\hline & \multicolumn{2}{|c|}{ Malla vial local existente } & \multicolumn{2}{|l|}{4264.10} & \\
\hline & \multicolumn{2}{|c|}{ Control ambiental existente } & \multicolumn{2}{|l|}{941.14} & \\
\hline
\end{tabular}

Fuente: Elaboración propia, bajo la recolección de datos en la técnica de análisis documental, basada en Alcaldía Mayor de Bogotá (2014). 
Como resultado del análisis de la información consignada en la tabla 2, el PPLS llega hasta la etapa de adopción sin haber definido concretamente con cuál UP operará; por su parte, el Decreto deja abierta la posibilidad de aplicar UAU o UG, esto depende de si existe o no consenso en la gestión de las UP.

\section{Plan Parcial De Renovación Urbana "San Victorino"}

Este PP opera en la modalidad de Redesarrollo, pretende avanzar mediante un desarrollo progresivo y expresa claramente que el instrumento de gestión a aplicar será la UAU. El área de ocupación de este PP se localiza a 300 metros del PPLS, es decir, está en la zona céntrica de la ciudad de Bogotá, por lo que es un sector de interés histórico y tradicional.

Para ser exactos, al norte delimita con la calle 13 (Av. Jiménez de Quezada); al sur, con la calle San Joaquín (calle 10); al oriente, con la Av. Fernando Mazuera (carrera 10a), y al occidente, con la Av. Caracas (carrera 14). En total son 14 manzanas catastrales: 10 correspondientes al barrio Santa Inés, y 4 establecidas en el barrio La Capuchina, pertenecientes a la Unidad de Planificación Zonal (UPZ) 93 Las Nieves, de la localidad de Santa Fe.

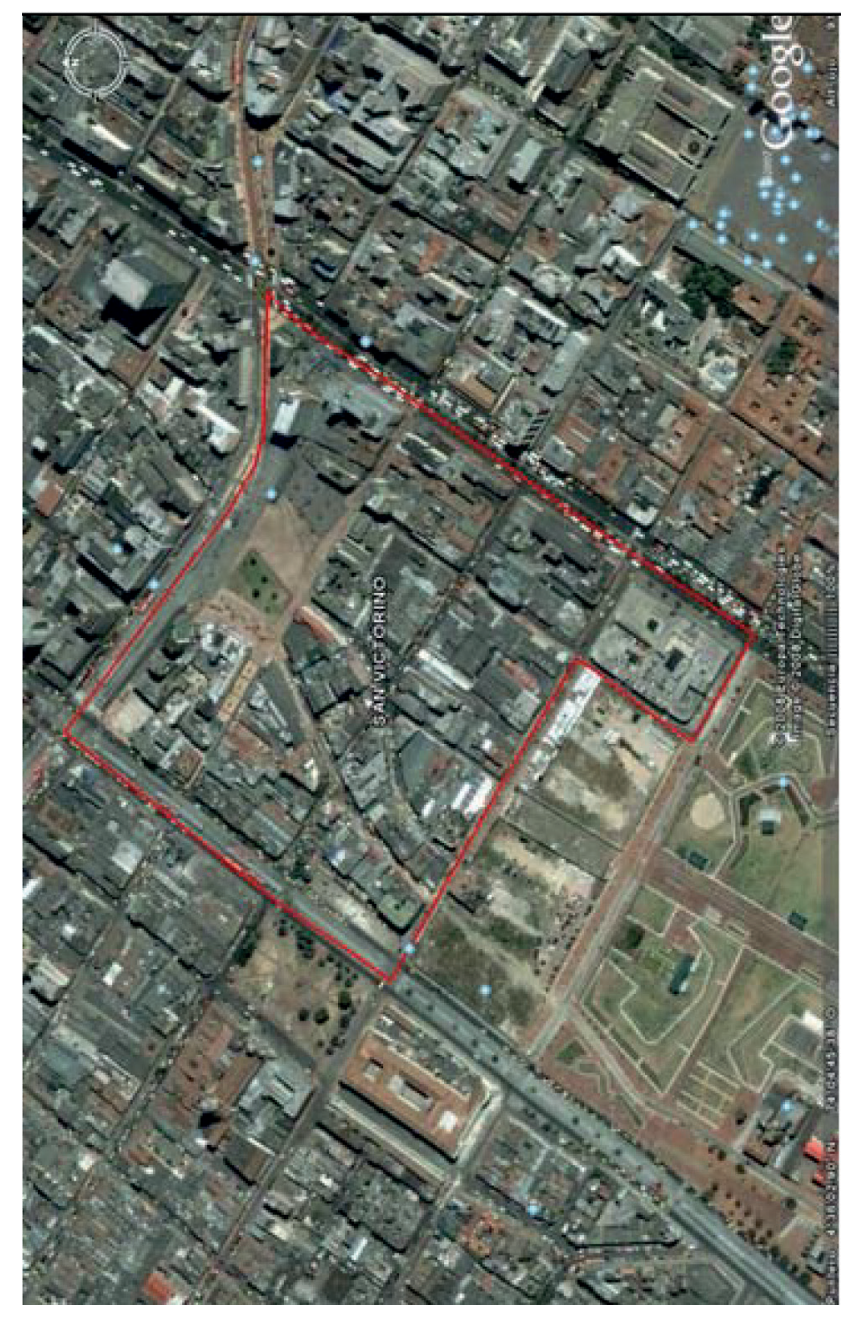

Figura 2. Localización del PPSV. Fuente: Secretaría Distrital de Planeación (s. f.). 
De acuerdo con la tabla 3, la zona de planificación de este PP cuenta con un área aproximada de $147000 \mathrm{~m}^{2}$ y está conformada por 12 manzanas a transformar (algunas de ellas tienen un alto grado de deterioro). La delimitación del área a intervenir se determinó considerando un modelo de ocupación adecuado a un comercio popular sostenible y teniendo en cuenta los estilos del sector, las costumbres y los usos desarrollados en el entorno más cercano (criterio similar al del PPLS, pero pensando también en la reconfiguración del paisaje). En total son 223 predios en las 12 manzanas vinculadas al proceso de planeación; sin embargo, en relación al uso comercial de algunas edificaciones que operan como centros comerciales, la variación de propiedades cambia sustancialmente por la existencia de 2928 locales.

Tabla 3. Ficha de caracterización del PPSV

\begin{tabular}{|c|c|c|c|c|c|}
\hline \multirow{7}{*}{ BASE PREDIAL } & N.o manzana & Área $\mathrm{m}^{2}$ & $\begin{array}{c}\text { Número de } \\
\text { predios }\end{array}$ & $\begin{array}{c}\text { Total } \\
\text { propiedades }\end{array}$ & $\begin{array}{c}\text { Total } \\
\text { propietarios }\end{array}$ \\
\hline & 23 & 5063.85 & 9 & 225 & \multirow{6}{*}{264} \\
\hline & 24 y $9 C$ & 5447.96 & 32 & 236 & \\
\hline & 1 y 8 & 10435.93 & 36 & 371 & \\
\hline & 2 y 91 & 4922.82 & 47 & 281 & \\
\hline & 16,20 y 21 & 19488.89 & 91 & 490 & \\
\hline & 27,28 & 11254.43 & 8 & 1325 & \\
\hline \multirow{9}{*}{ ÁREAS EXISTENTES M² } & \multicolumn{2}{|l|}{ Área bruta } & \multicolumn{3}{|c|}{147185.84} \\
\hline & \multicolumn{2}{|c|}{ Área neta urbanizable } & \multicolumn{3}{|c|}{67953.10} \\
\hline & \multicolumn{2}{|c|}{ Área útil } & \multicolumn{3}{|c|}{56365.92} \\
\hline & \multicolumn{2}{|c|}{ Malla vial arterial } & \multicolumn{3}{|c|}{21611.38} \\
\hline & \multicolumn{2}{|c|}{ Malla vial local existente } & \multicolumn{3}{|c|}{25308.70} \\
\hline & \multicolumn{2}{|c|}{ Control ambiental propuesto } & \multicolumn{3}{|c|}{5852.87} \\
\hline & \multicolumn{2}{|c|}{ Espacio público existente } & \multicolumn{3}{|c|}{17205.39} \\
\hline & \multicolumn{2}{|c|}{ Cesión espacio público } & \multicolumn{3}{|c|}{11552.04} \\
\hline & \multicolumn{2}{|c|}{ Cesión equipamiento público. } & \multicolumn{3}{|c|}{5416.06} \\
\hline
\end{tabular}

Fuente: Elaboración propia, bajo la recolección de datos en la técnica de análisis documental, basada en Alcaldía Mayor de Bogotá (2016).

El DTS del PPSV es muy preciso en cuanto a la selección de la UA con la que se pretende operar. Para este caso, el instrumento funcionaría con tan solo 2 unidades de actuación urbanística, que serían desarrolladas en 6 etapas de ejecución y estarían conformadas de la siguiente manera: en la UAU 1, la manzana 23 está en la etapa 1 de ejecución; 24 y 9, en la etapa 2; 1 y 8, en la etapa 3, y 2 y 9 , en la etapa 4. En esta unidad se asociará parte de la vivienda de interés social (VIS) y los equipamientos públicos. Además, actuará bajo el concepto de desarrollo prioritario definido en el artículo 40 de la Ley 388 del 97 y asociado a la declaratoria de utilidad pública e interés social contenido en el artículo 58 de la misma ley. De otro lado, en UAU 2, las manzanas 16, 20 y 21 están en la etapa 5, y las manzanas 27 y 28 , en la etapa 6 . Esta UAU se asocia a la construcción del remanente de VIS.

En consecuencia, queda entendido que este PP solo espera concluir su etapa de adopción y no contempla ninguna posibilidad de desarrollarse a través de las UG, toda vez que su territorio físico-espacial contiene un alto número de propiedades y se prevé que no habrá consenso en la gestión de las mismas. Razón por la que se propone un uso y aplicación de las UAU ligadas a la declaratoria de utilidad pública, sentido que marca la viabilidad del desarrollo prioritario propuesto según los criterios del artículo 39 del Decreto Distrital no 190 de 2004. 


\section{Plan Parcial De Renovación Urbana "La Felicidad"}

EL PPLF se ejecuta en una zona de tratamiento urbano en desarrollo, que consiste en un área urbanizable no urbanizada. Fue uno de los primeros $\mathrm{PP}$ formulados en el país y uno de los más grandes de Colombia. Opera en una de las zonas de mayor valorización de la ciudad, en cercanía a un sector que durante muchos años acogió una zona industrial y de bodegaje. La Felicidad, al noroccidente delimita con Modelia Sector E-Centro de Control EAAB-Zona de Servidumbre del Ferrocarril; al nororiente, con la Urbanización La Esperanza Etapa A; al suroriente, con la Avenida Boyacá; y al suroccidente, con la Avenida Centenario. El proyecto se ubica en un terreno sin manzanas y, hasta antes de su formulación, estaba ligado a los sectores Centenario, La Esperanza y barrio Modelia de la Unidad de Planificación Zonal (UPZ) 112 Granjas de Techo en la localidad de Fontibón.

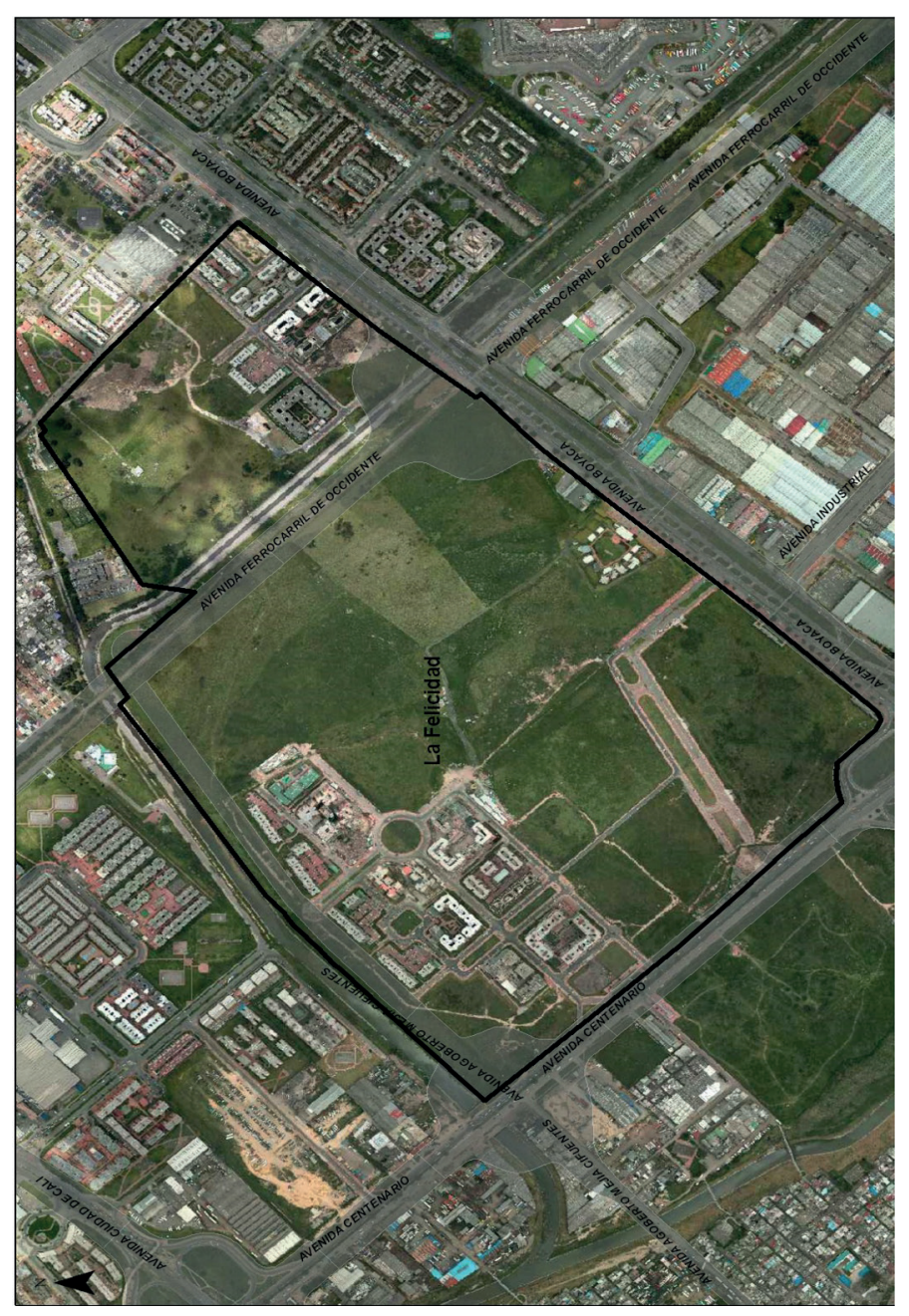

Figura 3. Localización del PPLF. Fuente: Secretaría Distrital de Planeación (2014).

La intervención planeada para este PP promete desarrollar un área bastante considerable, que en su extensión máxima llega aproximadamente a $1096000 \mathrm{~m}^{2}$, equivalentes a 109.6 hectáreas, en un terreno completamente libre para implantar y edificar. En la implementación de la Unidad de 
Planeamiento no fue necesario promover la unidad gestora para determinar la delimitación a través de las UAU, ya que este proyecto contó con el consenso de los tres propietarios, a saber, la sociedad MG S.A., Detergentes Ltda. y, posteriormente, la fábrica de grasas y productos químicos GRASCO S. A. Estos propietarios se encargaron de presentar la formulación del proyecto ante la Secretaria Distrital de Planeación y son responsables de aplicar la UG como instrumento de planificación de escala intermedia en este PP.

Tabla 4. Ficha de caracterización del PPLF

\begin{tabular}{|c|c|c|c|}
\hline & \multicolumn{2}{|c|}{ Área total delimitada } & 1092060.23 \\
\hline & \multicolumn{2}{|l|}{ Área bruta } & 999503.84 \\
\hline & \multicolumn{2}{|c|}{ Área neta urbanizable } & 870.000 .99 \\
\hline ÁREAS & \multicolumn{2}{|l|}{ Área útil } & 390866.30 \\
\hline EXISTENTES $\mathrm{m}^{2}$ & \multicolumn{2}{|c|}{ Malla vial intermedia y local } & 172588.60 \\
\hline & \multicolumn{2}{|c|}{ Control ambiental } & 33031.49 \\
\hline & \multicolumn{2}{|c|}{ Cesión espacio público } & 456020.92 \\
\hline & \multicolumn{2}{|c|}{ Equipamiento comunal público } & 67706.38 \\
\hline \multirow{6}{*}{$\begin{array}{c}\text { ETAPAS DEL } \\
\text { PLAN PARCIAL }\end{array}$} & Etapa & Área útil $\left(\mathrm{m}^{2}\right)$ & $\%$ de desarrollo \\
\hline & 1 & 43448 & $10.97 \%$ \\
\hline & 2 & 135863 & $34.30 \%$ \\
\hline & 3 & 86074 & $21.73 \%$ \\
\hline & 4 & 36282 & $9.16 \%$ \\
\hline & 5 & 94443 & $23.84 \%$ \\
\hline
\end{tabular}

Fuente: elaboración propia, bajo la recolección de datos en la técnica de análisis documental, basada en Alcaldía Mayor de Bogotá (2015).

E1 PP está distribuido en 54 manzanas, de las cuales 48 se destinan principalmente a vivienda. El PP se desarrolla a partir de 7 UG, que operan en 5 etapas de ejecución. El porcentaje de ejecución de la etapa 1 se fijó en 10.97 \%; la etapa 2, la más ambiciosa, en 34.30 \%; la etapa 3, en $21.73 \%$; la 4 , la más pequeña, en $9.16 \%$, y la etapa 5 , en $23.84 \%$.

Tal como se observa en la tabla 5, los predios de solo 3 propietarios (que son quienes formulan el plan) conforman el $98.56 \%$ de área total del proyecto, lo que representa un porcentaje suficiente para delimitar las UAU. Además, en este proyecto no se avista ningún propietario renuente, lo que permite la operación del PP mediante la aplicación de UG. En el DTS se advierte que:

Las Unidades de Gestión que no cumplan en su interior con las cesiones de suelo para componentes de la carga general, deberán cumplir con una de las siguientes condiciones: a) desarrollarse después de las unidades que tengan excedentes de cesiones y obligaciones que le permitan a las deficitarias cumplir;

b) entregar el suelo correspondiente, por fuera de la Unidad de Gestión, según el cuadro anterior;

c) ajustar la delimitación de la Unidad de Gestión para incluir cesiones y suelo útil para VIS que le permita cumplir con las áreas mínimas requeridas (DTS PPLF, 2015, p. 30).

El resultado de este análisis y la revisión de los documentos oficiales del PPLF demuestran que la posibilidad de formular un PP que operen con UG sí es viable, siempre y cuando exista el común acuerdo y en ningún momento se presenten propietarios renuentes, ya que ese factor marcaría y obligaría a una delimitación por UAU. Sin embargo, este proyecto no está exento del reparto 
UNIDADES DE ACTUACIÓN URBANISTICA O DE GESTIÓN: CASOS DE APLICACIÓN EN TRES PLANES PARCIALES DE BOGOTÁ D. C.

equitativo de cargas y beneficios, teniendo en cuenta que ese es otro instrumento determinante para el desarrollo de cualquier unidad de planeamiento.

Tabla 5. Porcentaje de injerencia de los Propietarios en los predios del PPLF

\begin{tabular}{|c|c|c|c|}
\hline $\begin{array}{c}\text { Folio de matrícula } \\
\text { inmobiliaria }\end{array}$ & $\begin{array}{c}\text { N.o propietarios y/o } \\
\text { poseedores }\end{array}$ & Área $\mathrm{m}^{2}$ & $\begin{array}{c}\% \text { de } \\
\text { participación }\end{array}$ \\
\hline $50 C-956982$ & 1 & 256.00 & \multirow{9}{*}{$1.43 \%$} \\
\hline $50 C-706841$ & 6 & 274.00 & \\
\hline $50 C-590112$ & 1 & 348.90 & \\
\hline $50 C-486440$ & 2 & 824.70 & \\
\hline $50 C-487992$ & 2 & 1068.40 & \\
\hline $50 C-1079810$ & 2 & 504.00 & \\
\hline $50 C-237549$ & 2 & 268.80 & \\
\hline $50 C-435249$ & 1 & 269.10 & \\
\hline $50 C-1084071$ & 1 & $\begin{array}{l}7800.32 \\
£=11614.00\end{array}$ & \\
\hline $\begin{array}{l}50 C-200881 \\
50 C-28164 \\
50 C-563575 \\
50 C-563576 \\
50 C-563577 \\
50 C-563578 \\
50 C-563579 \\
50 C-563580 \\
50 C-564316\end{array}$ & 1 & $\begin{array}{l}461824.00 \\
235573.00 \\
7056.97 \\
15041.76 \\
11136.18 \\
11136.16 \\
6532.34 \\
3786.07 \\
4915.75 \\
£=757002.00\end{array}$ & $92.96 \%$ \\
\hline $50 C-1420049$ & 1 & 11265.00 & $1.38 \%$ \\
\hline $\begin{array}{l}50 C-563574 \\
50 C-563573 \\
50 C-563572\end{array}$ & 1 & \begin{tabular}{|l|}
21948.00 \\
4592.80 \\
7869.12 \\
$£=34410.00$ \\
\end{tabular} & $4.22 \%$ \\
\hline TOTAL & & 814291.00 & $100 \%$ \\
\hline
\end{tabular}

Fuente: elaboración propia, bajo la recolección de datos en la técnica de análisis documental, basada en Alcaldía Mayor de Bogotá (2015).

\section{Conclusiones y RECOMENDACIONES}

Este análisis indica que la norma definió a las UAU como los instrumentos con los que es posible desarrollar cualquier actuación en el marco de los POT y, más explícitamente, de los PP, puesto que estos determinan la gestión de detalle de las áreas públicas y privadas. Además, se define cómo se va a aplicar el reparto de cargas y beneficios entre los diferentes actores que están involucrados en el proyecto, e incluso llega a exigir que se identifiquen las UAU que se van a desarrollar de manera prioritaria. Es decir, la Ley 388 solo señala las UAU como el instrumento idóneo de gestión para desarrollar los PP, junto con el reajuste de tierras o la integración inmobiliaria o cooperación entre participes, e incluso procesos de enajenación forzosa, como la expropiación.

Con el Decreto 2181 de 2006 aparece la UG, definida en el artículo 19, tal como se explicó en punto anterior. Según esta normativa, solo si todos los propietarios incluidos en la delimitación del PP están de acuerdo en la ejecución de la UA, se tramita una única licencia de urbanismo; en este 
caso, no se requiere la delimitación de UAU ante la administración. La diferencia radica básicamente en que no existan reparos al proyecto o que sea un solo propietario.

De acuerdo con lo expuesto anteriormente y con la intención de identificar los factores que determinan la aplicación y delimitación de las figuras analizadas, se puede deducir que un PP requiere de las UA para ejecutarse y esto solo es posible por intermedio de la UAU o la UG. De ahí que la Ley 388 del 97 señale que "una vez aprobado el plan parcial por la autoridad de planeación municipal o distrital, el proyecto de delimitación de las unidades de planeamiento es el siguiente paso" (art. 42), de lo que se infiere la necesidad de delimitar mediante estas unidades para el desarrollo del PP.

Lo que se debe hacer es delimitar la UAU, y una vez esté definida la entidad gestora para el desarrollo del proyecto se empieza a desarrollar el tema de gestión; momento en el que podría tomarse la decisión de operar el PP mediante UG, cumpliendo así con lo ya establecido, siempre y cuando no hayan propietarios renuentes; en caso de que estos existan, se debe adelantar el proceso de gestión en el marco de las UAU.

Se puede concluir que estructuralmente no hay diferencias entre las UAU y las UG, por cuanto las condiciones urbanísticas ya quedaron definidas en el decreto de PP. En consecuencia, las áreas públicas y privadas son inmodificables, bien sea por UAU o por UG. Adicionalmente, puede concluirse que la diferencia está en la gestión del suelo y del proyecto, es decir, esta diferencia es operativa, por eso se debe partir de UAU y luego ver si es posible lograr consenso para desarrollar el PP por UG.

En teoría, es posible que un PP pueda operar al mismo tiempo con las UAU y UG, pues generalmente los PP tienen más de una UAU y puede darse el caso que en algunas de ellas los propietarios se pongan de acuerdo, que exista el consenso y opten por UG. Pero también puede haber otras unidades afectadas por renuentes, por lo que se debe continuar solamente con UAU.

Las UAU y las UG son instrumentos inherentes a la gestión y operación de un PP. En consecuencia y después de confrontar diferentes factores en los PP comparados, cualquiera de las dos denominaciones es adecuada para la aplicación de estos instrumentos de desarrollo territorial de escala zonal. Los ámbitos estructurales se mantienen en cualquiera de los dos casos, y las condiciones de actuación de cada tipo de unidad solo cambia en un punto: cuando hay o no acuerdo total entre los propietarios.

\section{RefEREnCiAs}

Alcaldía Mayor de Bogotá. (2014). Documento técnico de soporte. Plan parcial La Sabana. Bogotá D.C: Alcaldía Mayor, Concreta Gestión Urbana S.A.S.

Alcaldía Mayor de Bogotá. (2015). Documento técnico de soporte. Plan parcial La Felicidad. Bogotá D. C.: Alcaldía Mayor, Inversiones MG S.A., Detergentes Ltda., GRASCO S.A.

Alcaldía Mayor de Bogotá. (2016). Documento Técnico de Soporte. Plan parcial San Victorino. Bogotá D.C: Alcaldía Mayor, Ecourbia.

Decreto 1212 de 2000. Por el cual se reglamenta el Acuerdo Municipal 062 de 1999, Plan de Ordenamiento Territorial de Medellin, en cuanto a los contenidos y procedimientos de los planes parciales. Alcaldía de Medellín, 5 de diciembre de 2000.

Decreto 2181 de 2006. Por el cual se reglamentan parcialmente las disposiciones relativas a planes parciales contenidas en la Ley 388 de 1997 y se dictan otras disposiciones en materia urbanistica. Diario Oficial 46320, 5 de julio de 2006 
UNIDADES DE ACTUACIÓN URBANISTICA O DE GESTIÓN: CASOS DE APLICACIÓN EN TRES PLANES PARCIALES DE BOGOTÁ D. C.

Decreto 147 de 2008. Por el cual se adopta el Plan Parcial "La Felicidad", ubicado en la Localidad de Fontibón. Alcaldía Mayor de Bogotá D. C., 19 de mayo de 2008.

Decreto 073 de 2015. Por el cual se adopta el Plan Parcial de Renovación Urbana "La Sabana", ubicado en la Localidad de Los Mártires y se dictan otras disposiciones. Alcaldía Mayor de Bogotá D. C., 26 de febrero de 2015.

Ley 388 de 1997. Por la cual se modifica la Ley 9a de 1989, y la Ley $3^{a}$ de 1991 y se dictan otras disposiciones. Diario Oficial 43091, 18 de julio de 1997.

Maldonado, M., Pinilla, J., Rodríguez, J., y Valencia, N. (2006) Planes parciales, gestión asociada y mecanismos de distribución equitativa de cargas y beneficios en el sistema urbanistico: marco jurídico, conceptos básicos y alternativas de aplicación. Bogotá, D. C.: Lincoln Institute of Land Policy.

Secretaría Distrital de Planeación. (s. f.). [Notificación a propietarios y posibles propietarios indeterminados del Plan Parcial Renovación Urbana "San Victorino"]. Recuperado de http:// www.sdp.gov.co/portal/page/portal/PortalSDP/OrdenamientoTerritorial/ArchivoPlanesParciales/PlanParcialSanVictorino/PPRU_San_victorino_Localizacion.pdf.

Secretaría Distrital de Planeación. (2014). Formulación-modificación del Plan Parcial "La Felicidad". Recuperado de http://www.sdp.gov.co/portal/page/portal/PortalSDP/OrdenamientoTerritorial/ArchivoPlanesParciales/Plan_Parcial_La_Felicidad/PLAN_PARCIAL_La_Felicidad_Diciembre_22_12_2014.pdf.

Tarchópulos, D. (2006) Las huellas del Plan para Bogotá de Le Corbusier, Sert y Wiener. Scripta Nova, 10(218). Recuperado de http://www.ub.edu/geocrit/nova.html. 